\title{
Acute renal failure in the cardiac intensive care unit - A practical approach to management
}

\section{Authors: Geoffrey Bihl MB.BCh M.MED FCP(SA), \\ Thomas Mabin MB.ChB FRCP, Derek Fine MD}

Geoffrey Bihl, MB.BCh M.MED FCP(SA)

Nephrologist, Winelands Kidney and Dialysis Centre, Somerset West

Thomas Mabin, MB.ChB FRCP

Cardiologist, Vergelegen Heart Unit, Somerset West

Derek Fine, MD

Associate Professor of Medicine

Division of Nephrology, Johns Hopkins School of Medicine, Baltimore, USA

\section{ABSTRACT The association between cardiovascular disease} and acute renal failure (ARF) and acute-on-chronic renal failure is less well defined. In the cardiac intensive care unit $(\mathrm{CICU})$, the presence of ARF imparts a significant mortality. The predominant aetiology of ARF in the $\mathrm{CICU}$ relates to hypotension, although other causes are certainly involved too, and both contrast nephropathy and cholesterol embolisation may be associated with percutaneous coronary intervention. Despite the availability of renal replacement therapies, preventative measures remain the most important element of management. Specific therapy includes management of the patient's volume status, vasoactive medication and renal replacement therapy in the way of dialysis. In the setting of acute renal failure, both patient and renal outcome is improved with the early involvement of a nephrological service.

\section{INTRODUCTION}

There is a strong and well-established association between cardiovascular disease and chronic kidney disease (CKD); however, the association between cardiovascular disease and acute renal failure (ARF) and acute-on-chronic renal failure is less well defined. What is clear is that, in the cardiac intensive care unit (CICU), the presence of ARF imparts a significant mortality, where up to $50 \%$ may die. Furthermore, the common association of diabetes and cardiovascular disease is further complicated by the concomitant risk of kidney disease. As the population ages, with its increasing comorbidities and acuity of illness, and the epidemic of cardiovascular disease continues to rise, we are likely to see more patients developing ARF in the $\mathrm{CICU}$ setting. Prevention and management of this development are of paramount importance to improve overall outcome of these patients and to practise cost-effective medicine.

Elderly patients are more likely to have comorbidities and more likely to develop renal failure, in association with percutaneous and surgical coronary interventions.
With judicious preventative strategies and aggressive management survival rates have improved, especially when adjusted for illness severity.(I)

\section{DEFINITION OF ACUTE RENAL FAILURE}

A widely accepted and systematic definition of ARF has been lacking, which has led to significant confusion both clinically and in the medical literature. In 2004, the Acute Dialysis Quality Initiative (ADQI) group published the RIFLE (Risk, Injury, Failure, Loss, End-stage kidney disease) classification of ARF, based on changes from the patient's baseline either in serum creatinine level or glomerular filtration rate (GFR), urine output (UO), or both. ${ }^{(2)}$ Although not universally accepted, we feel this provides an appropriate definition.

The RIFLE classification of ARF is as follows:

Risk (R) - Increase in serum creatinine level $X 1.5$ or decrease in GFR by $25 \%$, or $\cup O<0.5 \mathrm{~mL} / \mathrm{kg} / \mathrm{h}$ for 6 hours

Injury (I) - Increase in serum creatinine level $X 2.0$ or decrease in GFR by $50 \%$, or $\cup \bigcirc<0.5 \mathrm{~mL} / \mathrm{kg} / \mathrm{h}$ for 12 hours

Failure (F) - Increase in serum creatinine level $\times 3.0$, decrease in GFR by $75 \%$, or serum creatinine level $>350$ umol//; UO $<0.3 \mathrm{~mL} / \mathrm{kg} / \mathrm{h}$ for 24 hours, or anuria for 12 hours

Loss (L) - Persistent ARF, complete loss of kidney function $>4$ wk

End-stage kidney disease (E) - Loss of kidney function $>3$ months

Since baseline GFRs are not readily available, the consensus committee recommended the use of the Modification of Diet in Renal Disease (MDRD) equation to calculate the GFR. Most pathology laboratories in South Africa supply this calculation with the urea and electrolyte results.

\section{AETIOLOGIES OF ARF INTHE CICU}

Considering the common association between acute cardiac disease, hypotension and renal failure, it is not suprising that the prevailing aetiology of ARF in the $\mathrm{CICU}$ relates to hypotension. Other pathogenic mechanisms are involved, as outlined in (Figure I). 


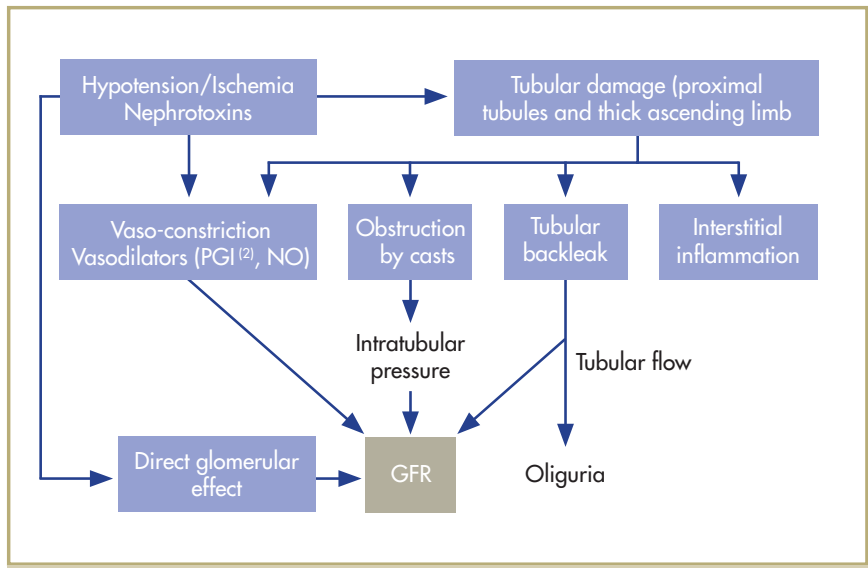

FIGURE 1: Pathophysiological mechanisms of $\mathrm{ARF}^{(35)}$

\section{Volume depletion, hypotension and cardiogenic shock}

Reduced cardiac output (CO) due to hypovolemia or cardiac dysfunction (acute MI, CHF, and arrhythmia) diminishes renal perfusion both directly and indirectly. Cardiogenic shock is a progressive state of hypotension (systolic blood pressure $<90 \mathrm{~mm} \mathrm{Hg}$ ) lasting at least 30 minutes, which leads to systemic hypoperfusion and is generally more common in patients with ST-segment elevation myocardial infarction than in patients with other acute coronary syndromes. ${ }^{(3)}$

Decreased $\mathrm{CO}$ lowers renal blood flow and activates a number of renal vasoconstrictor systems, which increase reno-vascular resistance (RVR). Neurohumoral responses (sympathetic nervous system, renin angiotensin aldosterone system (RAAS), vasopressin secretion) have opposing effects on renal perfusion, tending to augment renal perfusion pressure, but also resulting in renal vasoconstriction. Any intervention restoring $\mathrm{CO}$ and systemic perfusion therefore augments renal perfusion by reversing the aforementioned influences. ${ }^{(4)}$

The history in some patients often includes a decrease in fluid intake, vomiting and diarrhoea, haemorrhage, sepsis and/or excessive diuresis, all of which may have precipitated the acute cardiac event resulting in admission to the CICU.(5) Such patients may demonstrate a low jugular venous pressure, tachycardia, and postural hypotension. Kidney dysfunction associated with hypovolemia and low CO improves following volume replacement, inotropic support in those unresponsive to fluids, and improved myocardial function. Monitoring intravascular fluid status requires a combination of clinical parameters, central venous pressure, central venous oxygenation (via the CVP line), and lactate levels. However, sufficient improvement may be difficult to achieve in the setting of intractable myocardial dysfunction and/or cardiogenic shock where immediate revascularisation, where applicable, is associated with better outcome. ${ }^{(6)}$

\section{Congestive heart failure}

ARF is a common complication among patients hospitalized for $\mathrm{CHF}$, out of the setting of acute coronary ischemia, and is associated with increased risk for adverse outcomes. Approximately 50\% of patients hospitalised for heart failure have preserved systolic function; however, if their systolic blood pressure is less than $125 \mathrm{mmHg}$ their risk of acute renal failure increases, as does their mortality risk. (7) Risk factors for ARF from $\mathrm{CHF}$ include diabetes, elevated admission serum creatinine and reduced serum sodium and echocardiographic demonstration of diastolic dysfunction. ${ }^{(8)}$

\section{Percutaneous coronary intervention}

The two common causes of renal failure following $\mathrm{PCl}$ are contrast nephropathy (CN) and cholesterol embolisation (CE).

Cholesterol embolisation syndrome is a systemic disease caused by distal showering of cholesterol crystals after angiography, major vessel surgery, or thrombolysis. It is relatively rare following coronary intervention, and those patients with an elevated CRP pre-procedure may be at greater risk for its development. More than $60 \%$ of patients suffering from this syndrome develop renal insufficiency. ${ }^{(9)}$

Part of the difficulty in making the diagnosis relates to the time interval (typically one to four weeks but occasionally several months) between intervention and disease onset, which tends to obscure the causative link. In the context of renal disease, this delay in onset and subsequent progressive deterioration in renal function can help to distinguish cholesterol embolism from contrast nephropathy (see below). Given our inability to reverse the condition, early diagnosis is crucial if further episodes of cholesterol emboli are to be prevented. Statin therapy may stabilise plaques and a highly conservative approach, avoiding anticoagulation, angiography and vascular surgery, may produce the best outcome. Renal failure often progresses to dependence on dialysis. ${ }^{(10)}$

Importantly, acute renal failure that requires dialysis after percutaneous coronary interventions is associated with very high in-hospital and I-year mortality rates and a dramatic increase in hospital resource utilization. (II)

\section{$\mathrm{PCl}$ and contrast nephropathy}

Although definitions may vary, a commonly accepted and applied definition of contrast nephropathy is a > 25\% increase in serum creatinine level that occurs within 48 hours of contrast exposure. ${ }^{(12)}$ Once CN occurs, kidney function may remain depressed for I-3 weeks depending on the severity of the insult, but returns to normal or near normal in most cases.(13) The presence of predisposing risk factors (Table I) increases the risk of $\mathrm{CN}$ and, in the elderly patient undergoing coronary intervention, many risk factors may be present simultaneously. 
TABLE I: Strategies to reduce ARF

\begin{tabular}{l|ll}
\hline \multicolumn{1}{c}{ Proven } & \multicolumn{1}{c}{ Possible positve effects } & \multicolumn{1}{c}{ Ineffective } \\
\hline $\begin{array}{l}\text { Avoid nephrotoxins: } \\
\text { NSAIDS, Aminoglycosides }\end{array}$ & $\begin{array}{l}\text { Optimise renal perfusion: } \\
\text { Fluids and /or inotropic } \\
\text { support } \\
\text { N-acetylcysteine } \\
\text { (especially if using contrast) }\end{array}$ & $\begin{array}{l}\text { Low-dose dopamine } \\
\text { Mannitol } \\
\text { Diuretics } \\
\text { Aminophylline } \\
\text { Periprocedural dialysis }\end{array}$ \\
\hline $\begin{array}{l}\text { Modify drug dosage/volume: } \\
\text { contibiotics, nonionic }\end{array}$ & \\
\hline $\begin{array}{l}\text { Adequate Hydration: } \\
\text { Contrast agents } \\
\text { Tight glycemic control }\end{array}$ & \\
\hline
\end{tabular}

The most important risk factors seem to be the presence of preexisting kidney insufficiency, diabetes mellitus, fluid depletion, the elderly and the volume of contrast used. ${ }^{(14)}$ The use of nonionic agents ${ }^{(15)}$ appears to lower the incidence of $\mathrm{CN}$ and this has been confirmed in animal models. ${ }^{(16)}$

Recommendations for management of contrast nephropathy depend on the underlying kidney function.(17) In those patients with normal serum creatinine, adequate hydration is all that is necessary. Although serum creatinine levels may be normal, diabetics, elderly patients and those with reduced muscle mass may have a normal U\&E although their creatinine clearance may be reduced, thereby imparting a risk for $\mathrm{CN}$. In this setting, a calculated creatinine clearance would be more appropriate. In those with moderate kidney insufficiency (creatinine $150 \mathrm{mmol} / 1-250 \mathrm{mmol} / /$, creatinine clearance $<50 \mathrm{ml} / \mathrm{min}$ ) intravenous volume administration with saline $0.45 \%$ or $0.9 \%$ for 12 hours before and 6 hours after the procedure. Recent data suggests that isotonic sodium bicarbonate may be more protective, although this has not been substantiated. ${ }^{(18)}$ Iso-osmolar contrast agents with judicious dose limitation may also reduce the risk of CN and repeat procedures should be delayed as long as possible. Acetylcysteine $600 \mathrm{mg}$ bid on the day before and on the day of the procedure is advocated, though the benefit of this agent is controversial. Severe kidney insufficiency (creatinine $>250 \mathrm{mmol} / \mathrm{l}$, creatinine $<30 \mathrm{ml} / \mathrm{min}$ ) imparts a risk of irreversible kidney failure despite the abovementioned preventative measures. Such a risk should be discussed with the patient prior to the intervention and the need for the intervention should be debated.

Although the overall incidence of ARF after $\mathrm{PCl}$ is low, acute renal insufficiency after $\mathrm{PCl}$ is a strong and independent predictor of longterm mortality in patients who survive for 30 days after the procedure. ${ }^{(19)}$ Diabetic patients with baseline serum creatinine values $<170$ umol// are at higher risk than nondiabetic patients, whereas all patients with a serum creatinine $>170$ umol/l are at high risk for ARF. ${ }^{(20)}$

\section{Sepsis}

Although sepsis is a common cause of acute renal failure in the general intensive care unit, it is an unusual occurrence in the coronary care unit. Its presence as a primary disease, however, may precipitate an acute cardiac event.(21) The classical pathogenic mechanism of septic acute renal failure revolves around haemodynamic disease where fluid resuscitation aims to prevent and restore renal function. Recently, however, Wan et al. reviewed the subject and suggested that, indeed, acute tubular apoptosis may underlie the renal effects of sepsis, and management of precipitants may improve renal outcome. (22) Animal studies suggest that protein $\mathrm{C}$ has anti-inflammatory and anti-apoptotic functions, which are reduced in acute sepsis especially when acute renal failure is induced. In these studies treatment of septic animals with activated protein Cimproved renal function, suggesting anti-apoptosis. ${ }^{23}$ Mechanical ventilation causes pulmonary biotrauma, with a resultant pulmonary inflammatory reaction that may generate systemic release of inflammatory mediators, which have apoptotic effects, especially in the kidney. In theory, low volume mechanical ventilation may reduce such mediators and their renal side-effects. ${ }^{(24)}$ Intensive insulin therapy improves outcome in the ICU patient where strict glucose control seems to improve dysregulated apoptosis, which may aid in recovery from renal failure. ${ }^{(25,26)}$

Appropriate antibiotic usage adjusted for renal function and/or dialysis is equally important in controlling sepsis and its consequences.

\section{Medication}

Both cardiovascular disease and chronic kidney disease are frequently managed with ACE inhibitors, and there is strong evidence of better outcomes in both. However, ACE inhibitors can exacerbate or cause ARF in some settings. ACE inhibitor induced ARF occurs in settings where glomerular afferent arteriolar blood flow is reduced, in which case GFR is dependent on all mediated efferent arteriolar vasoconstriction. It should be stressed that ACE inhibitor induced ARF is not dependent on a drop in blood pressure or renal blood flow by the use of an ACE inhibitor. Indeed, in the presence of a significant renal artery stenosis, renal blood flow and GFR are blood pressure dependent and any intensification of antihypertensive treatment can cause reduced GFR. Having said that, subgroup analyses of the HOPE study indicate that ACE inhibition with ramipril is beneficial without an increased risk of adverse effects like acute renal failure or hyperkalemia. ${ }^{(27)}$ Thus the frequent practice of withholding ACE inhibitors from patients with mild renal insufficiency is unwarranted, especially as this might identify a group at high risk who appear to benefit most from treatment. There may be a place for acute withdrawal of the ACE inhibitor in the CCU setting when the patient is haemodynamically unstable or where the creatinine is rising acutely. However, the use of ACE inhibition 20 or more days post myocardial infarction has proven morbidity and 
mortality benefit and, although this may reduce glomerular pressure acutely with mild increase in serum creatinine, the serum creatinine then stabilises and in most cases returns to baseline.

Statins are used by millions of people worldwide and only rarely cause severe side effects, the most sinister of which is rhabdomyolysis induced ARF. To this end, the American Heart Association lists a group of patients most at risk of severe myopathy and these include: advanced age, multisystem disease (e.g. chronic renal failure, especially if caused by diabetes), perioperative periods, multiple medications (especially gemfibrozil, and cytochrome P3A4 interactors cyclosporine, itraconazole and ketoconazole,macrolide antibiotics,erythromycin and clarithromycin, HIV protease inhibitors in those on statins using this metabolic pathway) and alcohol abuse. ${ }^{(28)}$

Since the timing of this toxicity is unpredictable, routine screening is not recommended. Therefore, patients should be made aware of muscle symptoms and promptly discontinue the statin when these occur while awaiting laboratory (creatine kinase) assessment.

\section{MANAGEMENT STRATEGIES}

Despite the availability of renal replacement therapies, preventative measures remain the most important element of management. Table I outlines such therapies, although predicting who may be at risk for ARF, especially from contrast (Table 2), could circumvent its development.

TABLE 2: Risk factors for contrast nephropathy

Serum creatinine $>150 \mathrm{~mol} / \mathrm{l}$

2. Diabetic nephropathy

3. Older age

4. Class III/IV congestive heart failure

5. Volume and osmolality of contrast media used

Patients with acute cardiac events often have a preceding history of hypertension. This results in the autoregulatory level of renal blood flow being set at a higher level and renal blood flow decreases significantly when the mean arterial blood pressure falls below $80 \mathrm{mmHg}$, severely compromising renal perfusion and function.(29) These patients are at particular risk of hypoperfusion.

Reversal of a compromised haemodynamic state with inotropic support, appropriate fluid resuscitation and primary coronary intervention seems prudent in acutely compromised cardiac patients. Furthermore, removal of any nephrotoxic agents and precipitating factors are critical. Volume therapy is the cornerstone of initial management of the hypotensive patient and strict CVP monitoring is recommended. The rate and composition of the volume expanders is determined by the working diagnosis. Cardiogenic causes without evidence of fluid overload require small volume challenges $(250 \mathrm{ml})$ of crystalloid or perhaps hydroxyethyl starch titrated to clinical response (increased BP, decreased heart rate, increased urine output). Hypovolemic states require larger volumes of crystalloid, again titrated to response and underlying cardiac function. In this setting non-oliguric patients presenting with ARF have fewer complications, including a decreased dialysis requirement and improved survival. ${ }^{(30)}$ Although conversion of an oliguric patient to non-oliguria has less certain benefit, it can often be accomplished by repleting volume as above, and adding a loop diuretic titrated to urine output and blood pressure. An infusion of furosemide $250 \mathrm{mg}$ in $200 \mathrm{ml}$ normal saline can easily be adjusted to clinical response, but only once both blood pressure and central venous pressure are adequate.

Vasoactive therapy and monitoring should be simplified and goaldirected and reduced and discontinued when stability is achieved. ${ }^{(4)}$ The clinician must determine whether there is evidence of low cardiac output with high cardiac filling pressures that requires inotropic support or hypotension is accompanied by a high cardiac output state that requires pressor support. Generally speaking, beta agonists are chosen for support of cardiac output and alpha agonists are chosen when maintenance of perfusion pressure is needed to maintain blood flow to the tissues, including the kidneys. Common alpha agonists are phenylephrine and noradrenalin. Noradrenalin will raise mean arterial pressure at the expense of cardiac output and thus cardiac output should be adequate before its initiation. Dobutamine (beta-agonist) will increase cardiac output but will also vasodilate and thus venous return may need augmentation with a fluid bolus. Adrenaline has both alpha and beta effects and is used in resuscitation and severe hypotension. One must remember that long-term usage leads to tissue ischemia, the resultant acidosis of which may reduce effectiveness of other inotropic agents.

Importantly, whilst a renal vasodilator dose of dopamine (I.5 to $2.5 \mathrm{ug} / \mathrm{kg} / \mathrm{min}$ ) may stimulate urine volume, it does not improve GFR, shorten the duration of ARF, or decrease dialysis requirements. ${ }^{\left({ }^{(1)}\right)}$ In addition, dopamine may induce significant arrhythmia and possibly intestinal ischemia.

Many patients with ARF will recover renal function within days and not require renal replacement therapy (RRT). However, if the duration of ARF is prolonged, or if hyperkalemia, extracellular volume overload, refractory acidosis or uremic symptoms (serositis, encephalopathy, 
bleeding) occur, some form of renal replacement therapy may be necessary. The major goal of RRT in ARF is to maintain the patient's survival while awaiting recovery of renal function. Its two main objectives are I) to control ECV by removal of excess fluid (ultrafiltration) and 2) to control uremia by removal of excess solute (dialysis). Depending on a patient's needs, these two objectives can be accomplished separately or simultaneously, intermittently or continuously, by peritoneal or hematogenous access.

With no clear survival advantage established, the clinician is free to target a particular RRT modality to any given patient and, in fact, dialysis therapy should be individualised. ${ }^{(32)}$ When rapid solute control is necessary, e.g. severe hyperkalemia or volume overload, intermittent haemodialysis is most suitable. For hypotensive patients requiring inotropic support, continuous forms of dialysis such as continuous veno-veno haemodialysis may be more appropriate. They allow for more gentle fluid removal without further compromising the patient's haemodynamic status.

Regardless of the particular RRT selected, the clinician must ensure an adequate dose of dialysis. Currently, no measure of dialysis adequacy allows comparison of different RRTs applied to patients with ARF.(33)

Peritoneal dialysis (PD) plays a relatively minor role as an RRT in the CICU. Nevertheless, it offers several advantages including simplicity, hemodynamic stability and freedom from anticoagulation. Automated cyclers easily accomplish the exchange of dialysate; however, even with frequent dialysate exchanges and very hypertonic dialysate, PD clearance of solute and UF rates are limited. In addition, the intraperitoneal volume of dialysate can compromise respiration, and glucose loads and protein losses can be excessive. Therefore, PD appears better suited for the less catabolic, less uremic, and relatively normovolemic patient.

\section{CONCLUSION}

The development of ARF in the cardiac ICU patient portends an ominous outcome. Assessment and reduction of risk factors for ARF, thereby preventing its occurrence, is appropriate in all patients admitted and treated. When ARF does occur, early involvement of the kidney team (consultation and renal replacement) is likely to improve outcome in such patients. ${ }^{(34)}$

\section{REFERENCES:}

1. Bellomo R. The epidemiology of acute renal failure: 1975 versus 2005. Curr Opin Crit Care.2006; I2(6):557-60

2. Kellum JA, Bellomo R. The 3rd International Consensus Conference of the Acute Dialysis Quality Initiative (ADQI). Int J Artif Organs. 2005;28(5):44I-4

3. Holmes DR.Cardiogenic shock: a lethal complication of acute myocardial infarction.Rev Cardiovasc Med. 2003;4(3): | 31-5.

4. Holmes CL, Walley KR. Shock, in Intensive care in Nephrology. Murray PT, Brady HR eds Taylor and Francis.2007: 3-19.

5. Kumar A, Haery C.Myocardial dysfunction in septic shock. Crit Care Clin.2000; 16(2):25I-87.

6. Liistro F, Angioli P.Early invasive strategy in elderly patients with non-ST elevation acute coronary syndrome: comparison with younger patients regarding 30 day and long term outcome. Heart. 2005;9 I ( I0): 1284-8

7. Yancy CW, Lopitan N.Clinical presentation, management, and in-hospital outcomes of patients admitted with acute decompensated heart failure with preserved systolic function: a report from the Acute Decompensated Heart Failure National Registry (ADHERE) Database. I Am Coll Cardio.2006;47(1):76-84

8. Chittineni H, Fishbane S. Risk for acute renal failure in patients hospitalized for decompensated congestive heart failure. Am J Nephrol. 2007;27( I):55-62.

9. Y. Fukumoto, H. Tsutsui, M.The incidence and risk factors of cholesterol embolization syndrome, a complication of cardiac catheterization: a prospective study. J. Am. Coll. Cardiol.2003;42(2):21। - 216.

I0. Dupont PJ, Lightstone L.Cholesterol emboli syndrome. BMJ 2000;321:1065-1067.

11. Gruberg L, Mehran R.Acute renal failure requiring dialysis after percutaneous coronary interventions. Catheter Cardiovasc Interv. 200 I :52(4):409- 16

12. Porter G. Contrast medium-associated nephropathy. Invest Radiol. 1993;28(suppl. 4): $\mathrm{SII}-18$.

13. Berns AS. Nephrotoxicity of contrast media.Kidney Int. 1989:36:730-740

14. Rudnick MR, Kesselheim A. Contrast-induced nephropathy: how it develops, how to prevent it. Cleve Clin J Med. 2006 Jan;73( I):75-80, 83-7.

15. Soejima K, Uozumi J, Kanou T. Non-ionic contrast media are less nephrotoxic than ionic contrast media to rat renal cortical slices. Toxicol Lett.2003; I 43( I): 17-25.

16. Murphy SW, Barrett BJ, Parfrey PS. Contrast nephropathy. J Am Soc Nephrol. 2000; 1 1:177-82

17. Curhan GC. Prevention of contrast nephropathy. I Am Med Assoc.2003:289(5):606-608.

18. Merten G], Burgess WP. Prevention of contrast-induced nephropathy with sodium bicarbonate: a randomized controlled trial. JAMA. 2004:29I (19):2328-34.

19. Gupta R, Gurm HS. Renal failure after percutaneous coronary intervention is associated with high mortality. Catheter Cardiovasc Interv. 2005;64(4):442-8.

20. Rihal CS, Textor SC. Incidence and prognostic importance of acute renal failure after percutaneous coronary intervention.Circulation. 2002 ; 105(19):2259-64

21. Ensmiger SA, Wright RS.Suspected ventilator-associated pneumonia in cardiac patients admitted to the coronary care unit. Mayo Clin Proc. 2006:8 I (I):32-5.

22. Wan L, Bellomo R. The pathogenesis of septic acute renal failure. Curr Opin Crit Care. 2003 Dec;9(6):496-502

23. Gupta A, Berg DT, Gerlitz. Bole of protein C in renal dysfunction after polymicrobia sepsis. J Am Soc Nephrol. 2007 Mar; 18(3):860-7.

24. Kuiper JW, Groeneveld AB, Slutsky AS, Plotz FB. Mechanical ventilation and acute renal failure. Crit Care Med. 2005;33(6): 1 408-15.

25. Van der Berghe G,Wouters P. Intensive insulin therapy in the critically ill patients. N Eng Med. 200 I;345(19):1359-67.

26. Wesche-Soldato DE, Swan RZ, Chung CS, Ayala A. The apoptotic pathway as therapeutic target in sepsis. Curr Drug Targets. $2007 ; 8(4): 493-500$.

27. Mann JF, Yi QL. Serum potassium, cardiovascular risk, and effects of an ACE inhibitor: results of the HOPE study. Clin Nephrol. 2005;63(3): I 81-7.

28. Pasternak RC, Smith SC Ir. American College of Cardiology; American Heart Association; National Heart, Lung and Blood Institute. ACC/AHA/NHLBI Clinical Advisory on the Use and Safety of Statins. Circulation. 2002; 106: 1024-1028.

29. Palmer BF. Renal dysfunction complicating the treatment of hypertension. N Engl J Med. 2002 Oct 17;347(|6):|256-6|

30. Anderson RJ, Linas SL. Nonoliguric acute renal failure. N Engl | Med. 1977:296(20): | I 34- I 138.

31. Bellomo R, Chapman M. Low-dose dopamine in patients with early renal dysfunction: a placebo-controlled randomised trial. Australian and New Zealand Intensive Care Society (ANZICS) Clinical Trials Group. Lancet. 23-30 2000:356(9248):2139-2I 43.

32. Mehta RL, Macdonald B. A randomized clinical trial of continuous versus intermittent dialysis for acute renal failure. Kidney Int. 200 I;60(3): I I54-63.

33. Evanson JA, Himmelfarb J, Wingard R.Prescribed versus delivered dialysis in acute renal failure patients. Am J Kidney Dis. 1998;32(5):731-738.

34. Acute renal failure in the cardiac care unit: etiologies, outcomes, and prognostic factors. Kidney Int. 1999;56( I):238-43.

35. Finkel KW, Murray PT. Prevention of acute renal failure in the intensive care unit. In, Intensive care in Nephrology. Murray PT, Brady HR eds. Taylor and Francis.2007:71-98. 